\title{
Abnormal Gastric Myoelectrical Activity and Delayed Gastric Emptying in Asthmatics: What Is Clinical Significance?
}

TO THE EDITOR: I read with interest the study by Amarasiri et $\mathrm{al}^{1}$ regarding the relationship between gastric dysmotility and asthma using electrogastrography (EGG) and real time ultrasonography. The study was conducted by enrolling 30 asthmatics and 30 healthy volunteers. The study reported that asthmatics showed abnormal gastric myoelectrical activity (GMEA), delayed gastric emptying (GE) and antral hypomotility in response to a solid meal, compared to healthy controls. Abnormal GMEA especially during the postprandial period is associated with severe gastroesophageal reflux disease (GERD) or delayed GE. ${ }^{2}$ Delayed GE contributes to a progressive dilatation of the proximal stomach which, in turn, shortens the length of the lower esophageal sphincter until it becomes competent. ${ }^{3}$ This is why delayed GE can promote gastroesophageal reflux episodes, and then can provoke the exacerbation in asthmatics. I would like to consider 2 concerns for the accurate understanding of the meaning of these results.

First, the clinical significance of abnormal GMEA was not clear. It must be noted that EGG does not diagnose a specific disease, even the stomach motor dysfunctions. ${ }^{4}$ The ranges of $\%$ normal waves, dominant frequency, pre/postprandial power ratio were also within normal range, despite of the significant differences between the 2 groups. However, the normal range of GMEA observed in asthmatics should not be devaluated because GE is the final coordination of fundus, antrum, pylorus and duodenum, and then normal EGG parameters do not guarantee normal GE
Finally, delayed GE observed in asthmatics group might have resulted from the high proportion of GERD among asthmatics. In other words GERD might be confounding factor between the delayed GE and asthma. This is because delayed GE is frequent in patients with GERD. ${ }^{5}$

Further larger study with well-designed methodology is needed to confirm whether abnormal GMEA, delayed GE and antral hypomotility are significantly more prevalent in asthmatics.

Tae Hee Lee

Institute for Digestive Research Soonchunhyang University, College of Medicine, Seoul, Korea

1. Amarasiri WA, Pathmeswaran A, de Silva AP, Dassanayake AS, Ranasinha CD, de Silva HJ. Gastric motility following ingestion of a solid meal in a cohort of adult asthmatics. J Neurogastroenterol Motil 2013;19:355-365.

2. Cucchiara S, Salvia G, Borrelli O, et al. Gastric electrical dysrhythmias and delayed gastric emptying in gastroesophageal reflux disease. Am J Gastroenterol 1997;92:1103-1108.

3. Pellegrini CA. Delayed gastric emptying in patients with abnormal gastroesophageal reflux. Ann Surg 2001;234:147-148.

4. Chang FY. Electrogastrography: basic knowledge, recording, processing and its clinical applications. J Gastroenterol Hepatol 2005;20: 502-516.

5. Emerenziani S, Sifrim D. Gastroesophageal reflux and gastric emptying, revisited. Curr Gastroenterol Rep 2005;7:190-195.

\section{Conflicts of interest: None.}

